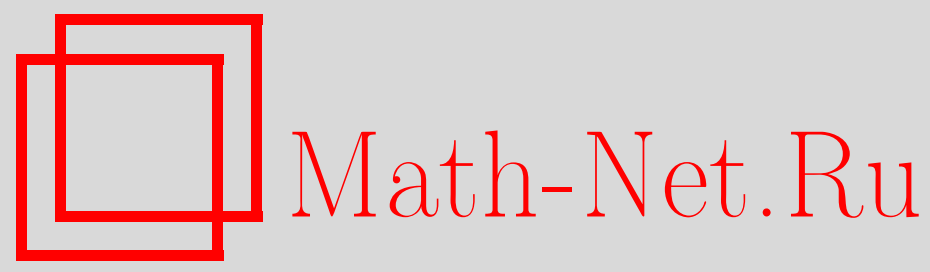

О. К. Иванова, Мажорантные оценки для частичных сумм кратных рядов Фурье функций из пространств Орлича, равных нулю на некотором множестве, Матем. заметки, 1999, том 65, выпуск 6, 821-830

DOI: https://doi.org/10.4213/mzm1118

Использование Общероссийского математического портала Math-Net.Ru подразумевает, что вы прочитали и согласны с пользовательским соглашением http://www.mathnet.ru/rus/agreement

Параметры загрузки:

IP : 18.234 .197 .8

26 апреля 2023 г., $15: 26: 43$

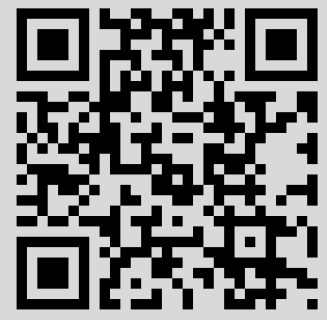




\section{МАЖОРАНТНЫЕ ОЦЕНКИ ДЛЯ ЧАСТИЧНЫХ СУММ КРАТНЫХ РЯДОВ ФУРЬЕ ФУНКЩИЙ ИЗ ПРОСТРАНСТВ ОРЛИЧА, РАВНЫХ НУЛЮ НА НЕКОТОРОМ МНОЖКСТВЕ}

\section{О.К. Иванова}

В работе рассматриваются $\sup _{n}\left|S_{n}(x ; f)\right|$ - мажоранты частичных сумм двойных тригонометрических рядов Фурье функций из $L\left(\log ^{+} L\right)^{3}$, равных 0 на некоторых подмножествах $T^{2}=[-\pi, \pi)^{2}$. В частности, доказывается

Tеорема. Пусть $f(x) \in L\left(\log ^{+} L\right)^{3}\left(T^{2}\right)$ u $f(x)=0$ на $T^{2} \backslash Q$, где $Q-\kappa в а \partial-$ рат со сторонами, параллельными координатным осям $\left(Q \subset T^{2}\right)$. Тогда для любого $\delta>0$ существует константа $C_{\delta}>0$ такая, что

$$
\left\|\sup _{n}\left|S_{n}(x ; f)\right|\right\|_{L_{1}\left(T_{\delta}^{2} \backslash Q\right)} \leqslant C_{\delta} \iint_{Q}|f(x)|\left(\log ^{+}|f(x)|\right)^{3} d x+C_{\delta},
$$

әде $T_{\delta}^{2}=\left\{x \in \mathbb{R}^{2}:-\pi+\delta \leqslant x_{j} \leqslant \pi-\delta, j=1,2\right\}, C_{\delta}$-константа, не зависящая ни от функиии $f$, ни от квадрата $Q$.

Библиография: 15 названий.

1. Рассмотрим $N$-мерное евклидово пространство $\mathbb{R}^{N}$, элементы которого будем обозначать через $x=\left(x_{1}, \ldots, x_{N}\right)$, и положим $n x=n_{1} x_{1}+\cdots+n_{N} x_{N}$.

Для любого $\lambda \in \mathbb{R}^{1}$ определим множество $\mathbb{R}_{\lambda}^{N}=\left\{x \in \mathbb{R}^{N}: x_{j} \geqslant \lambda, j=1, \ldots, N\right\}$. Кроме того, введем множество $\mathbb{Z}^{N} \subset \mathbb{R}^{N}$ всех векторов с целочисленньми координатами и положим $\mathbb{Z}_{\lambda}^{N}=\mathbb{Z}^{N} \cap \mathbb{R}_{\lambda}^{N}$.

Пусть $\Phi:[0, \infty) \rightarrow[0, \infty)$ - неубьвающая функция, через $\Phi(L)=\Phi(L)\left(T^{N}\right)$ обозначим множество суммируемых функций $f$ таких, что

$$
\int_{T^{N}} \Phi(|f(x)|) d x<\infty
$$

где $T^{N}=\left\{x \in \mathbb{R}^{N}:-\pi \leqslant x_{j}<\pi, j=1, \ldots, N\right\}$. Если $\Phi(u)=u^{p}$, то $\Phi(L)=L_{p}\left(T^{N}\right)$, $p \geqslant 1$, если $\Phi(u)=u \log ^{+} u$, где $\log ^{+} u=\log \max \{1, u\}$, то $\Phi(L)=L \log ^{+} L$.

Работа выполнена при финансовой поддержке Российского фонда фундаментальных исследований, грант № 96-01-00332, а также при поддержке проекта "Ведущие научные пшколы РФ”, грант № 96-15-96073. 
Пусть $2 \pi$-периодическая (по каждому аргументу) функция $f(x) \in \Phi(L)\left(T^{N}\right), N \geqslant 1$, разложена в кратный тригонометрический ряд Фурье:

$$
f(x) \sim \sum_{k \in \mathbb{Z}^{N}} c_{k} e^{i k x}
$$

Для любого вектора $n=\left(n_{1}, \ldots, n_{N}\right) \in \mathbb{Z}_{0}^{N}$ рассмотрим прямоугольную частичную сумму этого ряда

$$
S_{n}(x ; f)=\sum_{\left|k_{1}\right| \leqslant n_{1}} \cdots \sum_{\left|k_{N}\right| \leqslant n_{N}} c_{k} e^{i\left(k_{1} x_{1}+\cdots+k_{N} x_{N}\right)}
$$

частным случаем которой является квадратная частичная сумма $S_{n_{0}}(x ; f)$, когда $n_{1}=$ $n_{2}=\cdots=n_{N}=n_{0}$.

При этом под сходимостью ряда в (1) по прямоугольникам будем понимать существование предела частичных сумм (2) при $n \rightarrow \infty$, т.е. при $\min _{1 \leqslant j \leqslant N} n_{j} \rightarrow \infty$, a под сходимостью ряда в (1) по квадратам - существование предела $S_{n_{0}}(x ; f)$ при $n_{0} \rightarrow \infty$.

Пусть $\Omega$ - произвольное измеримое множество, $\Omega \subset T^{N}, N \geqslant 1,0<\mu \Omega<(2 \pi)^{N}$ ( $\mu=\mu_{N}$ есть $N$-мерная мера Лебега), и пусть $f(x)=0$ на $\Omega$.

2. Для кратных рядов Фурье И. Л. Блошанским было введено понятие обобщенной локализации почти всюду (см. [1], [2]).

ОПРЕДЕЛЕниЕ. Пусть $\Omega \subset T^{N}$ - произвольное множество положительной меры. Будем говорить, что для кратных рядов Фурье функций из класса $\Phi(L)\left(L_{\infty}\right)$ справедлива на множестве $\Omega$ обобщенная локализация почти всюду, если из условия $f \in$ $\Phi(L)\left(T^{N}\right)\left(f \in L_{\infty}\left(T^{N}\right)\right), f(x)=0$ на $\Omega$, следует, что почти всюду на $\Omega$ существует предел

$$
\lim _{n \rightarrow \infty} S_{n}(x ; f)=0
$$

В работах [1], [2] был доказан обобщенньй принцип локализации почти всюду для двойных тригонометрических рядов Фурье, которьй можно сформулировать, в частности, в следующем виде (см. также [3]).

ТЕОремА А. Пусть $\Omega \subset T^{2}$ - произвольное (непустое) открытое множество и пусть $f(x) \in L_{p}\left(T^{2}\right), p>1, f(x)=0$ на $\Omega$. Тогда

1) $\lim _{n \rightarrow \infty} S_{n}(x ; f)=0$ для почти всех $x \in \Omega$;

2) для любого $\varepsilon>0$ существуют открытое множество $\Omega_{\varepsilon} \subset \Omega, \mu \Omega_{\varepsilon}>\mu \Omega-\varepsilon$, и постоянная $C(\varepsilon, p)$ такие, что

$$
\left\|\sup _{n \in \mathbb{Z}_{0}^{2}}\left|S_{n}(x ; f)\right|\right\|_{L_{p}\left(\Omega_{\varepsilon}\right)} \leqslant C(\varepsilon, p)\|f\|_{L_{p}\left(T^{2}\right)} .
$$


Таким образом, из теоремы А следует, что обобщенная локализация почти всюду справедлива на любых открытых почти всюду множествах $\Omega \subset T^{2}$ для двойных рядов Фурье функций из классов $L_{p}\left(T^{2}\right), p>1$. Множество $\Omega$ назовем открытым почти всюд $y$, если существует открытое множество $E$ такое, что $\mu(\Omega \Delta E)=0$.

Обзор результатов, показьвающих существенность ограничений в теореме $\mathrm{A}(N=2$, $p>1, \Omega$ - открытое множество), см., например, в [4]-[6].

Результаты теоремы А (в частности, оценка (3)) привели к новому вопросу. Существуют ли открытые почти всюду множества $\Omega \subset T^{2}$ такие, что мажорантная оценка (3) справедлива на всем множестве $\Omega$ ? Другими словами, влияет ли геометрия открытого почти всюду двумерного множества $\Omega$ на мажорантную оценку (3)?

Пусть $Q \subset T^{2}$ - квадрат со сторонами, параллельными координатным осям, т.е.

$$
Q=[a, a+q] \times[b, b+q]
$$

где $a, b, q \in \mathbb{R}^{1}, q>0$.

В работе [7] Блошанским была доказана (см. [7, лемма 3])

Tеорема Б. Пусть $f(x) \in L_{p}\left(T^{2}\right), p>1$, и nусmь $f(x)=0$ на $T^{2} \backslash Q$. Тогда

$$
\left\|\sup _{n \in \mathbb{Z}_{0}^{2}}\left|S_{n}(x ; f)\right|\right\|_{L_{p}\left(T^{2} \backslash Q\right)} \leqslant C(p)\|f\|_{L_{p}(Q)}
$$

где квадрат $Q$ определен в (4), константа $C(p)$ не зависит ни от функиии $f$, ни от квадрата $Q$.

Как отмечалось в работе [7, с. 255], результат теоремы Б остается справедливым, если вместо квадрата (4) рассмотреть прямоугольник $\left[a, a+q_{1}\right] \times\left[b, b+q_{2}\right] \subset T^{2}$ или объединение конечного числа таких прямоугольников (в последнем случае константа $C(p)$ в (5) будет зависеть от числа прямоугольников).

В работах [8]-[10] продолжено исследование обобщенной локализации почти всюду на открытых множествах $\Omega \subset T^{2}$ в классах $\Phi(L)\left(T^{2}\right)$. В частности, в этих работах доказаны следующие результаты, обобщающие теорему А для функций из классов, “лежаших между" $L_{1}$ и $L_{p}, p>1$, равных 0 на открытом множестве $\Omega$.

ТЕОремА В. Пусть $\Omega \subset T^{2}$ - произвольное (непустое) открытое множество $u f(x)=0$ на $\Omega$. Тогда

1) если $f \in L \log ^{+} L \log ^{+} \log ^{+} L\left(T^{2}\right)$, mo

$$
\lim _{n \rightarrow \infty} S_{n}(x ; f)=0 \quad \text { для почти всех } \quad x \in \Omega
$$

2) если $f \in L\left(\log ^{+} L\right)^{2}\left(T^{2}\right)$, то для любого $\varepsilon>0$ существуют открытое множество $\Omega_{\varepsilon} \subset \Omega, \mu \Omega_{\varepsilon}>\mu \Omega-\varepsilon$, и постоянная $C_{\varepsilon}>0$ такие, ито

$$
\left\|\sup _{n \in \mathbb{Z}_{0}^{2}}\left|S_{n}(x ; f)\right|\right\|_{L_{1}\left(\Omega_{\varepsilon}\right)} \leqslant C_{\varepsilon} \int_{T^{2}}|f(x)|\left(\log ^{+}|f(x)|\right)^{2} d x+C_{\varepsilon} .
$$


Таким образом, обобшенная локализация почти всюду справедлива при $N=2$ на открытых почти всюду множествах для функций из классов $L \log ^{+} L \log ^{+} \log ^{+} L\left(T^{2}\right)$; в свою очередь, мажорантная оценка на подмножестве $\Omega_{\varepsilon}$ множества $\Omega$ (сколь угодно мало отличающемся от всего множества $\Omega$ ) справедлива уже для функций из класca $L\left(\log ^{+} L\right)^{2}\left(T^{2}\right)$.

3. Естественно возникает такой вопрос: для функций из какого класса $\Phi(L)\left(T^{2}\right)$, $\Phi(u)=o\left(u^{p}\right), p>1, u \rightarrow \infty$, будет справедлива оценка вида (5).

В настоящей работе мы даем следующий ответ на этот вопрос.

Teорема. Пусть $f(x) \in L\left(\log ^{+} L\right)^{3}\left(T^{2}\right)$ u $f(x)=0$ на $T^{2} \backslash Q$. Тогда для любого $\delta>0$ существует константа $C_{\delta}>0$ такая, что

$$
\left\|\sup _{n \in \mathbb{Z}_{0}^{2}}\left|S_{n}(x ; f)\right|\right\|_{L_{1}\left(T_{\delta}^{2} \backslash Q\right)} \leqslant C_{\delta} \iint_{Q}|f(x)|\left(\log ^{+}|f(x)|\right)^{3} d x+C_{\delta}
$$

әде $T_{\delta}^{2}=\left\{x \in \mathbb{R}^{2}:-\pi+\delta \leqslant x_{j} \leqslant \pi-\delta, j=1,2\right\}$, квадрат $Q$ определен в (4), константа $C_{\delta}$ не зависит ни от функиии $f$, ни от квадрата $Q$.

ЗАмечАниЕ 1. Так же, как теорема Б, сформулированная теорема остается справедливой, если вместо квадрата (4) рассмотреть прямоугольник $\left[a, a+q_{1}\right] \times\left[b, b+q_{2}\right]$, где $a, b, q_{1}, q_{2} \in \mathbb{R}^{1},\left[a, a+q_{1}\right],\left[b, b+q_{2}\right] \subset T^{2}$, или объединение конечного числа таких прямоугольников (в последнем случае константа $C$ в $(6)$ будет зависеть от числа прямоугольников).

ЗАмЕчАниЕ 2. В основе доказательства сформулированной теоремы лежит конструкция, предложенная Блошанским для доказательства теоремы Б (см. [7, лемма 3]).

Далее, естественно возникает вопрос о справедливости оценки (6) в “более широком", чем $L\left(\log ^{+} L\right)^{3}$ классе.

Очевидно, что оценка (6) несправедлива в тех классах $\Phi(L)$, где обобщенная локализация почти всюду несправедлива на открытых множествах. В частности, учитьвая результат работы [10, теорема 2], оценка (6) несправедлива в любом классе $\Phi(L)$, где $\Phi(u)=o\left(u \log ^{+} \log ^{+} u\right)$ при $u \rightarrow \infty$.

4. ДоКАЗАТЕЛЬСТВО ТЕОРЕМЫ. Отметим, что при доказательстве мы будем рассматривать и оценивать не частичную сумму $S_{n, m}(x, y ; f)$, т.е. оператор

$$
S_{*}(x, y ; f)=\sup _{n, m>0}\left|S_{n, m}(x, y ; f)\right|
$$

а оператор

$$
J_{*}\left(x, y ; f_{1}\right)=\sup _{n, m>0}\left|J_{n, m}\left(x, y ; f_{1}\right)\right|,
$$

где

$$
J_{n, m}\left(x, y ; f_{1}\right)=\frac{1}{\pi^{2}} \iint_{T^{2}} f_{1}(u, v) \frac{\sin n(u-x)}{u-x} \frac{\sin m(v-y)}{v-y} d u d v
$$


- собственный интеграл Фурье функции

$$
f_{1}(x, y)= \begin{cases}f(x, y) & \text { при }(x, y) \in T^{2} \\ 0 & \text { вне } T^{2}\end{cases}
$$

Мы можем себе это позволить, так как в [8] установлено, что для любой функции $f \in$ $L\left(\log ^{+} L\right)^{2}\left(T^{2}\right)$ и любого $\delta>0$ существует постоянная $C_{\delta}>0$ такая, что

$$
\left\|R_{*}(x, y ; f)\right\|_{L_{1}\left(T_{\delta}^{2}\right)} \leqslant C_{\delta} \iint_{T^{2}}|f(x)|\left(\log ^{+}|f(x)|\right)^{2} d x+C_{\delta},
$$

где $T_{\delta}^{2}=\left\{x \in \mathbb{R}^{2}:-\pi+\delta \leqslant x_{j} \leqslant \pi-\delta, j=1,2\right\}$,

$$
R_{*}(x, y ; f)=\sup _{n, m>0}\left|R_{n, m}(x, y ; f)\right|=\sup _{n, m>0}\left|S_{[n],[m]}(x, y ; f)-J_{n, m}\left(x, y ; f_{1}\right)\right|
$$

$n, m \in \mathbb{R}^{1},[n]$ - целая часть $n$ (см. [8, теорема 3$\left.]\right)$.

Итак, рассмотрим $J_{*}\left(x, y ; f_{1}\right)$. В силу определения функции $f$, а следовательно, и $f_{1}$ можем записать $J_{n, m}\left(x, y ; f_{1}\right)$ в виде

$$
J_{n, m}\left(x, y ; f_{1}\right)=\frac{1}{\pi^{2}} \iint_{Q} f(u, v) \widetilde{D}_{n}(u-x) \widetilde{D}_{m}(v-y) d u d v
$$

где $\widetilde{D}_{l}(z)=(\sin l z) / z-$ упрошенное ядро Дирихле. Полагая $P=T^{2} \backslash Q$ и учитывая $(7),(8)$, имеем

$$
\begin{aligned}
J\left(f_{1}\right) & =\iint_{P} \sup _{n, m>0}\left|\frac{1}{\pi^{2}} \iint_{Q} f_{1}(u, v) \widetilde{D}_{n}(u-x) \widetilde{D}_{m}(v-y) d u d v\right| d x d y \\
& =\iint_{P} J_{*}\left(x, y ; f_{1}\right) d x d y=\iint_{P_{1}+\cdots+P_{8}} J_{*}\left(x, y ; f_{1}\right) d x d y=\sum_{k=1}^{8} \mathfrak{J}_{k}\left(f_{1}\right),
\end{aligned}
$$

где $P_{1}, \ldots, P_{8}$ определяются следующим образом. Так как квадрат (4) образован прямыми, делящими $T^{2}$ на девять частей, включая сам квадрат $Q$, то это означает, что множество $P=T^{2} \backslash Q$ состоит из восьми прямоугольников. Занумеруем эти прямоугольники по часовой стрелке, начиная с левого нижнего прямоугольника. Таким образом, учитьвая (4), имеем

$$
\begin{gathered}
P_{1}=[-\pi, a] \times[-\pi, b], \quad P_{2}=[-\pi, a] \times[b, b+q], \quad P_{3}=[-\pi, a] \times[b+q, \pi], \\
P_{4}=[a, a+q] \times[b+q, \pi], \quad P_{5}=[a+q, \pi] \times[b+q, \pi], \\
P_{6}=[a+q, \pi] \times[b, b+q], \quad P_{7}=[a+q, \pi] \times[-\pi, b], \quad P_{8}=[a, a+q] \times[-\pi, b] .
\end{gathered}
$$

Оценим интегралы $\mathfrak{J}_{k}\left(f_{1}\right), k=1, \ldots, 8$. Интегралы $\mathfrak{J}_{1}\left(f_{1}\right), \mathfrak{J}_{3}\left(f_{1}\right), \mathfrak{J}_{5}\left(f_{1}\right), \mathfrak{J}_{7}\left(f_{1}\right)$ оцениваются одинаково; в свою очередь, получение оценок для $\mathfrak{J}_{2}\left(f_{1}\right), \mathfrak{J}_{6}\left(f_{1}\right), \mathfrak{J}_{4}\left(f_{1}\right)$, $\mathfrak{J}_{8}\left(f_{1}\right)$ также совпадает. Оценим по одному интегралу из каждой группы. 
Лемма 1. Пусть $k=1,3,5,7$. Тогда

$$
\mathfrak{J}_{k}\left(f_{1}\right) \leqslant C \iint_{Q}|f(u, v)|\left(\log ^{+}|f(u, v)|\right)^{2} d u d v+C,
$$

где $C$ - константа.

ДокАЗАТЕльСТво проведем для $k=1$. Для остальных $k$ доказательство аналогично. Очевидно, что

$$
\begin{aligned}
\mathfrak{J}_{1}\left(f_{1}\right) & =\frac{1}{\pi^{2}} \iint_{P_{1}} \sup _{n, m>0}\left|\iint_{Q} f_{1}(u, v) \widetilde{D}_{n}(u-x) \widetilde{D}_{m}(v-y) d u d v\right| d x d y \\
& \leqslant \frac{1}{\pi^{2}} \int_{-\pi}^{a} \int_{-\pi}^{b} \int_{a}^{a+q} \int_{b}^{b+q} \frac{\left|f_{1}(u, v)\right|}{|u-x| \cdot|v-y|} d u d v d x d y \\
& =\frac{1}{\pi^{2}} \int_{0}^{a+\pi} \int_{0}^{b+\pi} \int_{0}^{q} \int_{0}^{q} \frac{\left|f_{1}(u+a, v+b)\right|}{(u+x)(v+y)} d u d v d x d y \\
& \leqslant \frac{1}{\pi^{2}} \int_{0}^{A} \int_{0}^{A} \int_{0}^{A} \int_{0}^{A} \frac{\left|f_{1}(u+a, v+b)\right|}{(u+x)(v+y)} d u d v d x d y \\
& =\frac{1}{\pi^{2}} \int_{0}^{A} \int_{0}^{A} \frac{1}{u+x} \int_{0}^{A} \int_{0}^{A} \frac{\left|f_{1}(u+a, v+b)\right|}{v+y} d u d v d x d y
\end{aligned}
$$

(мы сделали замену переменных $u^{\prime}=u-a, v^{\prime}=v-b, x^{\prime}=a-x, y^{\prime}=b-y$ и положили $A=\max \{a+\pi, b+\pi, q\})$.

Далее, рассмотрим оператор

$$
(G \varphi)(z)=\int_{0}^{\infty} \frac{\varphi(t)}{t+z} d t, \quad z \in \mathbb{R}_{0}^{1}
$$

для функций $\varphi(t) \in L \log ^{+} L\left(\mathbb{R}_{0}^{1}\right)$.

ПРЕДЛОЖЕНИЕ. Пусть $\varphi(t) \in L \log ^{+} L\left(\mathbb{R}_{0}^{1}\right)$. Тогда справедливо неравенство

$$
\int_{0}^{\infty}|(G \varphi)(z)| d z \leqslant C \int_{0}^{\infty}|\varphi(t)| \log ^{+}|\varphi(t)| d t+C,
$$

әде константа $C$ не зависит от функиии $\varphi$, а оператор $G \varphi$ определен в (12).

ДокАЗАТЕЛЬСТво. Нам понадобится следующая теорема С. Яно [11] (см. также [12, c. 179-180], распространенная $\Phi$. Сориа [13] на линейные операторы, действующие из $L_{r}(X)$ в $L_{r}(X), 1<r<\infty$, с мерой $\left.\mu X=\infty\right)$. 
ТЕОРема Г. Пусть оператор $h=G \varphi$ имеет сильный mun $(r, r)$ для каждого $r$, $1<r<\infty$, maк что

$$
\|h\|_{L_{r}\left(\mathbb{R}_{0}^{1}\right)} \leqslant B_{r}\|\varphi\|_{L_{r}\left(\mathbb{R}_{0}^{1}\right)}, \quad 1<r<\infty .
$$

Тогда если

$$
B_{r}=O\left(\frac{1}{r-1}\right), \quad r \rightarrow 1,
$$

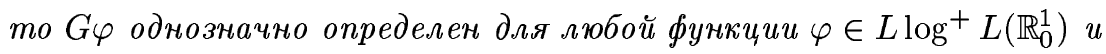

$$
\int_{0}^{\infty}|h| d x \leqslant C \int_{0}^{\infty}|\varphi| \log ^{+}|\varphi| d x+C
$$

где константа С не зависит от $\varphi$.

Учитывая, что для оператора $G$ из (12) справедливо неравенство Гильберта-ШураХарди (см. [14, с. 318-319])

$$
\|G \varphi\|_{L_{r}\left(\mathbb{R}_{0}^{1}\right)} \leqslant B_{r}\|\varphi\|_{L_{r}\left(\mathbb{R}_{0}^{1}\right)}, \quad 1<r<\infty
$$

где константа $B_{r}$ имеет следующую асимптотику (по $r$ ):

$$
B_{r}=\int_{0}^{\infty}(y+1)^{-1} y^{-1 / r} d y=\frac{\pi}{\sin \pi(1-1 / r)}=O\left(\frac{1}{r-1}\right) \quad \text { при } r \rightarrow 1,
$$

получаем (см. теорему Г, оценки (14) и (15)) оценку (13). Предложение доказано.

Далее, применяя в (11) предложение сначала к функции

$$
\varphi_{1}(v)=\varphi_{1}(v ; \cdot)= \begin{cases}\left|f_{1}\left(u_{0}+a, v+b\right)\right|, & v \in[0, A], \\ 0, & v \in(A, \infty),\end{cases}
$$

а затем к функции

$$
\varphi_{2}(u)=\varphi_{2}(u ; \cdot)= \begin{cases}\left|f_{1}\left(u+a, v_{0}+b\right)\right| \log ^{+}\left|f_{1}\left(u+a, v_{0}+b\right)\right|, & u \in[0, A], \\ 0, & u \in(A, \infty),\end{cases}
$$

можем оценить $\mathfrak{J}_{1}\left(f_{1}\right)$ следуюшим образом:

$$
\begin{aligned}
\mathfrak{J}_{1}\left(f_{1}\right) \leqslant & C \int_{0}^{A} \int_{0}^{A} \frac{1}{u+x}\left(\int_{0}^{A}\left|f_{1}(u+a, v+b)\right| \log ^{+}\left|f_{1}(u+a, v+b)\right| d v+C\right) d u d x \\
= & C \int_{0}^{A} \int_{0}^{A} \int_{0}^{A} \frac{\left|f_{1}(u+a, v+b)\right| \log ^{+}\left|f_{1}(u+a, v+b)\right|}{u+x} d u d x d v+C \\
\leqslant & C \int_{0}^{A}\left(\int_{0}^{A}\left|f_{1}(u+a, v+b)\right| \log ^{+}\left|f_{1}(u+a, v+b)\right|\right. \\
& \left.\times \log ^{+}\left(\left|f_{1}(u+a, v+b)\right| \log ^{+}\left|f_{1}(u+a, v+b)\right|\right) d u+C\right) d v+C \\
\leqslant & C \int_{0}^{A} \int_{0}^{A}\left|f_{1}(u+a, v+b)\right|\left(\log ^{+}\left|f_{1}(u+a, v+b)\right|\right)^{2} d u d v+C \\
= & C \iint_{Q}\left|f_{1}(u, v)\right|\left(\log ^{+}\left|f_{1}(u, v)\right|\right)^{2} d u d v+C .
\end{aligned}
$$

Лемма 1 доказана. 
Лемма 2. Пусть $k=2,4,6,8$. Тогда

$$
\mathfrak{J}_{k}\left(f_{1}\right) \leqslant C \iint_{Q}|f(u, v)|\left(\log ^{+}|f(u, v)|\right)^{3} d u d v+C
$$

где $C$ - константа.

ДокАЗАТЕЛьСТво проведем для $k=2$. Для остальных $k$ доказательство аналогично. Очевидно, что

$$
\mathfrak{J}_{2}\left(f_{1}\right)=\frac{1}{\pi} \iint_{P_{2}} \sup _{n, m>0}\left|\frac{1}{\pi} \iint_{Q} f_{1}(u, v) \widetilde{D}_{n}(u-x) \widetilde{D}_{m}(v-y) d u d v\right| d x d y
$$

Учитьвая вид множества $P_{2}$, можем оценить $\mathfrak{J}_{2}\left(f_{1}\right)$ следующим образом:

$$
\mathfrak{J}_{2}\left(f_{1}\right) \leqslant \frac{1}{\pi} \int_{b}^{b+q} \int_{-\pi}^{a} \sup _{m>0} \int_{a}^{a+q}\left|\frac{1}{\pi} \int_{b}^{b+q} f_{1}(u, v) \widetilde{D}_{m}(v-y) d v\right| \frac{d u}{|u-x|} d x d y
$$

Следовательно, полагая

$$
J_{*}\left[F_{u}(y)\right]=\sup _{m>0}\left|J_{m}\left[F_{u}(y)\right]\right|
$$

где

$$
J_{m}\left[F_{u}(y)\right]=\frac{1}{\pi} \int_{b}^{b+q} f_{1}(u, v) \widetilde{D}_{m}(v-y) d v
$$

можем записать

$$
\begin{aligned}
\mathfrak{J}_{2}\left(f_{1}\right) & \leqslant \frac{1}{\pi} \int_{b}^{b+q} \int_{-\pi}^{a} \int_{a}^{a+q} \frac{J_{*}\left[F_{u}(y)\right]}{|u-x|} d u d x d y \\
& =\frac{1}{\pi} \int_{b}^{b+q} \int_{0}^{a+\pi} \int_{0}^{q} \frac{J_{*}\left[F_{u+a}(y)\right]}{u+x} d u d x d y \\
& \leqslant \frac{1}{\pi} \int_{0}^{A} \int_{0}^{A} \frac{1}{u+x} \int_{0}^{A} J_{*}\left[F_{u+a}(y)\right] d y d u d x
\end{aligned}
$$

(мы сделали замену переменных $u^{\prime}=u-a, x^{\prime}=x-a$ и положили $A=\max \{b+q$, $a+\pi, q\})$.

Учитывая результат П. Шелина [15] и равномерную равносходимость разложений в одномерный ряд и интеграл Фурье (см. [12, с. 362-363]), получаем (см. также [8])

$$
\left\|\sup _{m>0}\left|J_{m}(x ; \varphi)\right|\right\|_{L_{1}\left(T^{1}\right)} \leqslant C \int_{T^{1}}|\varphi|\left(\log ^{+}|\varphi|\right)^{2} d x+C
$$

где $J_{m}(x ; \varphi)$ - собственньй интеграл $\Phi$ урье, определенньй формулой $(8)$ для одномерного случая $T^{1}=[-\pi, \pi]$. 
Далее, в силу (18) и (17) имеем

$$
\begin{aligned}
\mathfrak{J}_{2}\left(f_{1}\right) & \leqslant C \int_{0}^{A} \int_{0}^{A} \frac{1}{u+x}\left(\int_{0}^{A}\left|f_{1}(u+a, v)\right|\left(\log ^{+}\left|f_{1}(u+a, v)\right|\right)^{2} d v+C\right) d u d x \\
& =C \int_{0}^{A} \int_{0}^{A} \int_{0}^{A} \frac{\left|f_{1}(u+a, v)\right|\left(\log ^{+}\left|f_{1}(u+a, v)\right|\right)^{2}}{u+x} d v d u d x+C \\
& =C \int_{0}^{A} \int_{0}^{A} \int_{0}^{A} \frac{\left|f_{1}(u+a, v)\right|\left(\log ^{+}\left|f_{1}(u+a, v)\right|\right)^{2}}{u+x} d u d x d v+C .
\end{aligned}
$$

Применяя в (19) предложение к функции

$$
\varphi_{3}(u)=\varphi_{3}(u ; \cdot)= \begin{cases}\left|f_{1}\left(u+a, v_{0}+b\right)\right|\left(\log ^{+}\left|f_{1}\left(u+a, v_{0}+b\right)\right|\right)^{2}, & u \in[0, A] \\ 0, & u \in(A, \infty)\end{cases}
$$

оценим $\mathfrak{J}_{2}\left(f_{1}\right)$ следующим образом:

$$
\begin{aligned}
\mathfrak{J}_{2}\left(f_{1}\right) \leqslant & C \int_{0}^{A}\left(\int_{0}^{A}\left|f_{1}(u+a, v)\right|\left(\log ^{+}\left|f_{1}(u+a, v)\right|\right)^{2}\right. \\
& \left.\times \log ^{+}\left(\left|f_{1}(u+a, v)\right|\left(\log ^{+}\left|f_{1}(u+a, v)\right|\right)^{2}\right) d u+C\right) d v+C \\
\leqslant & C \int_{0}^{A} \int_{0}^{A}\left|f_{1}(u+a, v)\right|\left(\log ^{+}\left|f_{1}(u+a, v)\right|\right)^{3} d u d v+C \\
= & C \iint_{Q}\left|f_{1}(u, v)\right|\left(\log ^{+}\left|f_{1}(u, v)\right|\right)^{3} d u d v+C .
\end{aligned}
$$

Лемма 2 доказана.

Таким образом, лемма 1 (оценка (10)), лемма 2 (оценка (16)) и формула (9) доказывают оценку (6). Теорема доказана.

В заключение автор выражает глубокую благодарность И. Л. Блошанскому за постановку задачи и постоянное внимание к работе.

\section{СПИСОК ЦИТИРОВАННОЙ ЛИТЕРАТУРЫ}

[1] Блошанский И. Л. Равносходимость разложений в кратный тригонометрический ряд Фурье и интеграл Фурье // Матем. заметки. 1975. Т. 18. № 2. С. 153-168.

[2] Блошанский И. Л. Обобщенная локализация почти всюду и сходимость двойных рядов Фурье // Докл. АН СССР. 1978. Т. 242. №1. С. 11-13.

[3] Блошанская С. К., Блошанский И. Л., Рослова Т. Ю. Обобщенная локализация в классах Орлича // Современные проблемы теории функций и их приложения. Тезисы докл. 9-й Саратовской зимней школы, 1989. Саратов, 1997. С. 26.

[4] Bloshanskii I. L. Generalized localization and convergence tests for double trigonometrie Fourier series of functions from $L_{p}, p>1 / /$ Anal. Math. 1981. V. 7. №1. P. 3-36. 
[5] Блошанский И. Л. Автореферат дисс. ... д. ф.-м. н. М.: МИАН, 1991.

[6] Блошанский И. Л. Структура и геометрия максимальных множеств сходимости и неограниченной расходимости почти всюду кратных рядов Фурье функций из $L_{1}$, равных нулю на заданном множестве // Изв. АН СССР. Сер. матем. 1989. Т. 53. № 4. С. 675-707.

[7] Блошанский И. Л. Два критерия слабой обобщенной локализации для кратных тригонометрических рядов Фурье функций из $L_{p}, p \geqslant 1 / /$ Изв. АН СССР. Сер. матем. 1985. Т. 49. № 2. C. 243-282.

[8] Блошанский И.Л., Иванова О.К., Рослова Т. Ю. Обобщенная локализация и равносходимость разложений в двойной тригонометрический ряд и интеграл Фурье функций из $L\left(\ln ^{+} L\right)^{2} / /$ Матем. заметки. 1996. Т. 60. № 3. С. 437-441.

[9] Рослова Т. Ю. О справедливости обобщенной локализации для двойных тригонометрических рядов Фурье функций из $L \log ^{+} L \log ^{+} \log ^{+} L / /$ Докл. РАН. 1998. Т. 359. №6. C. $744-745$.

[10] Блошанская С.К., Блошанский И. Л., Рослова Т. Ю. Обобщенная локализация для двойных тригонометрических рядов Фурье и рядов Фурье-Уолша функций из $L \log ^{+} L \times$ $\log ^{+} \log ^{+} L / /$ Матем. сб. 1998. T. 189. № 5. C. 21-46.

[11] Yano S. Notes on Fourier analysis (XXIX): An extrapolation theorem // J. Math. Soc. Japan. 1951. V. 3. P. 296-305.

[12] Зигмунд А. Тригонометрические ряды. Т. 2. М.: Мир, 1965.

[13] Soria F. Note on differentiation of integrals and the halo conjecture // Studia. Math. 1985. V. 81. P. 29-36.

[14] Стейн И. Сингулярные интегралы и дифференциальные свойства функций. М.: Мир, 1973.

[15] Sjolin P. Convergence almost everywhere of certain singular integrals and multiple Fourier series // Ark. Mat. 1971. V. 9. №1. P. 65-90.

Московский государственный технологический университет "Станкин"

Поступило

25.06 .98

Исправленный вариант

24.11 .98 